\title{
Tetrandrine inhibits the proliferation of human osteosarcoma cells by upregulating the PTEN pathway
}

\author{
DONG-DONG TIAN ${ }^{1,2}$, RAN-XI ZHANG ${ }^{1,2}$, NIAN WU ${ }^{1,2}$, WEI YUAN ${ }^{1}$, SHUAI-HONG LUO ${ }^{1}$, HOU-QING CHEN ${ }^{1}$, \\ YANG LIU $^{1}$, YANG WANG ${ }^{1}$, BAI-CHENG HE ${ }^{2,3}$ and ZHONG-LIANG DENG ${ }^{1}$ \\ ${ }^{1}$ Department of Orthopaedics, The Second Affiliated Hospital, ${ }^{2}$ Chongqing Key Laboratory of Biochemistry \\ and Molecular Pharmacology, ${ }^{3}$ Department of Pharmacology, School of Pharmacy, \\ Chongqing Medical University, Yuzhong, Chongqing 400010, P.R. China
}

Received November 6, 2016; Accepted January 16, 2017

DOI: $10.3892 /$ or.2017.5560

\begin{abstract}
Tetrandrine (TET) is a natural product isolated from the Chinese herb Stephania tetrandra S. Moore and has been reported to have antiproliferation and apoptosis-inducing activity in various malignant tumor cells. However, the exact molecular mechanisms underlying these effects remain unclear. In the present study, we tested the antiproliferation effect of TET on osteosarcoma (OS) 143B cells and explored the possible potential molecular mechanism in this process. Using CCK-8 assay and flow cytometry, we found that TET inhibited proliferation, induced apoptosis and arrested the cell cycle of the 143B cells. Using a xenograft tumor model of human OS, tetrandrine was found to inhibit tumor growth in vivo. TET increased the protein level of phosphatase and tensin homolog (PTEN) and decreased its phosphorylation as detected by western blot analysis and immunohistochemistry. Overexpression of PTEN strengthened the anticancer effect of TET, while knockdown of PTEN attenuated it. Meanwhile, TET activated p38 MAPK and increased its phosphorylation. Our findings suggest that TET may be a potential anticancer drug for OS. In addition, its effects may be mediated by the upregulation of PTEN. Moreover the expression alteration of PTEN and p-PTEN was mediated by the TET-induced activation of p38 MAPK in a direct or indirect manner.
\end{abstract}

\section{Introduction}

As a malignant bone tumor, osteosarcoma (OS) is most prevalent in children and young adults. It accounts for $\sim 2.4$ and $20 \%$ of all pediatric cancers and primary bone malignancies, respectively (1-3). The current treatment of

Correspondence to: Professor Zhong-Liang Deng, Department of Orthopaedics, The Second Affiliated Hospital, Chongqing Medical University, 76 Linjiang Road, Yuzhong, Chongqing 400010, P.R. China

E-mail: zhongliang.deng@yahoo.com; zhongliang.deng@qq.com

Key words: osteosarcoma, tetrandrine, antiproliferation, PTEN, p38 MAPK
OS is mainly based on surgery and chemotherapy, and the treatment outcomes are limited (4). Chemotherapy drugs, such as methotrexate, doxorubicin, cisplatin, oxaliplatin (OXA), 5-fluorouracil (5-FU), bleomycin, Taxol and etoposide are not effective and even possess numerous side-effects and toxicities $(5,6)$. Therefore, understanding the molecular mechanisms of OS cells is highly beneficial, and more efficient and novel pharmaceutical products for OS are pressingly needed. Recently, traditional Chinese medicine (TCM) has successfully gained people's attention. Moreover, a large number of plant-derived bioactive compounds used in TCM are now used to treat different types of malignant cancer.

As a natural product extracted from Chinese herbs, tetrandrine (TET) [(1b)-6,6',7,12-tetramethoxy-2,2'-dimethyl-berbaman] is a bisbenzylisoquinoline alkaloid isolated from the Chinese herb Stephania tetrandra S. Moore (7). TET has been used for years as a clinical drug in China to treat patients with silicosis, autoimmune disorders, inflammatory pulmonary and cardiovascular diseases, and hypertension (8). Recently, evidence has indicated that TET possesses an antitumor effect through its antiproliferative and apoptosis-inducing abilities in various malignant cancers, such as gastric (9), bladder (10), prostate (11) and colon cancer (12), gallbladder carcinoma (13) and OS (14). Tao et al reported that TET induces the apoptosis of human OS cells through the activation of the mitochondrial pathway (15). However, there are still few studies exploring the possible mechanisms underlying the TET-induced antiproliferation and apoptosis in OS cells.

Identified as a tumor-suppressor gene, phosphatase and tensin homolog (PTEN) functions as a lipid phosphatase. PTEN dephosphorylates phosphatidylinositol $(3,4,5)$-triphosphate $\left(\mathrm{PIP}_{3}\right)$, and counteracts the activity of phosphatidylinositol-3-kinase (PI3K). PI3K phosphorylates phosphatidylinositol $(4,5)$-bisphosphate $\left(\mathrm{PIP}_{2}\right)$ to generate $\mathrm{PIP}_{3}$. In addition, $\mathrm{PIP}_{3}$ is the membrane anchor and ligand of the pleckstrin homology (PH) domain of AKTs (16). Moreover, PTEN negatively regulates not only PI3K signaling, but also mitogen-activated protein kinases (MAPKs), which are downstream of RAS (17). Deletions or the inactivation of mutations of PTEN are observed in a large number of various cancers at high frequency. MAPK consist of a family of protein kinases 
such as ERK, JNK and p38, which phosphorylate specific serines and threonines of target protein substrates and regulate cellular activities including gene expression, mitosis, motility, metabolism and programmed death (18). Among the MAPKs family, it has been recently found that p38 MAPK plays a significant role in the induction of apoptosis in various cell systems (19).

It has been recently reported that p38 MAPK and PTEN can affect each other mutually in numerous types of cancer. For example, Li et al reported that the phosphorylated form of PTEN enhances p38 signaling (20), while Wu et al reported that p38 MAPK enhances PTEN signaling (21). However, to date, there is no study addressing the relationship between p38 MAPK and PTEN in OS. We hypothesized that p38 MAPK may interact with PTEN in the TET-induced antiproliferation process in OS. In addition, our data ascertained that TET can inhibit the proliferation of OS cells, and this process may be mediated by the upregulation of PTEN through at least in part the activation of $\mathrm{p} 38$ MAPK.

\section{Materials and methods}

Agent preparations and cell culture. TET (purity $=99.2 \%$ ) was obtained from Hao-Xuan Bio-Technology Co., Ltd. (Xi'an, China), and was dissolved in dimethyl sulfoxide (DMSO) for in vitro experiments as well as prepared with $0.4 \%$ carboxymethylcellulose sodium (CMC-Na) for in vivo experiments. Antibodies were obtained from Santa Cruz Biotechnology (Santa Cruz, CA, USA). The p38 inhibitor SB203580 (\#S1076) was purchased from Selleckchem (Houston, TX, USA). The human OS cell line 143B was purchased from the American Type Culture Collection (ATCC; Manassas, VA, USA). Cells were cultured in Dulbecco's modified Eagle's medium (DMEM) with $10 \%$ fetal bovine serum (FBS), $100 \mathrm{U} / \mathrm{ml}$ of penicillin and $100 \mu \mathrm{g} / \mathrm{ml}$ of streptomycin at $37^{\circ} \mathrm{C}$ in $5 \% \mathrm{CO}_{2}$.

Cell Counting Kit-8 (CCK-8) assay. Sub-confluent 143B cells were seeded into 96-well plates (150 $\mu \mathrm{l}, 1,500$ cells/well) and cultured for $24 \mathrm{~h}\left(37^{\circ} \mathrm{C}, 5 \% \mathrm{CO}_{2}\right)$. Then, cells were treated with pre-designated concentrations $(1,2,3,4$ and $5 \mu \mathrm{M})$ of TET, recombinant adenovirus or DMSO. After 24,48 and $72 \mathrm{~h}$, CCK-8 (10 $\mu 1 /$ well; 7Sea Biotech, Shanghai, China) was added to the cells, and the 96-well plate was continuously incubated for $2 \mathrm{~h}$. Subsequently, the OD value for each well was read at a wavelength of $450 \mathrm{~nm}$ to determine the cell viability on a microplate reader. Each condition was carried out in triplicate. The cell viability was calculated as follows:

$$
\text { Cell viability }(\%)=\frac{\mathrm{OD}(\text { experiment })-\mathrm{OD} \text { (blank) }}{\mathrm{OD}(\text { control })-\mathrm{OD}(\text { blank })} \times 100
$$

Construction of recombinant adenovirus. Recombinant adenoviruses expressing PTEN (AdPTEN) and small interfering RNA fragments targeting PTEN (AdsiPTEN) were constructed with the AdEasy system (22). AdPTEN and AdsiPTEN were tagged with red fluorescence protein (RFP). The recombinant adenovirus expression RFP alone was used as a vector control. There were 4 fragments targeting PTEN in AdsiPTEN, and each of the fragments top line sequence is as follows: AGCTAAAGGTGAAGATATA; AGTAAGGACCA

\section{GAGACAAA; CAGATAATGACAAGGAATA; AGAAAGA} CTTGAAGGCGTA.

Flow cytometric analysis for apoptosis and cell cycle. Sub-confluent 143B cells were seeded into 6-well plates, and treated with pre-designated concentrations $(1,2$ and $4 \mu \mathrm{M})$ of TET, recombinant adenovirus or DMSO for $24 \mathrm{~h}$. For the cell cycle analysis, cells were harvested and washed with phosphatebuffered saline (PBS) $\left(4^{\circ} \mathrm{C}\right)$, collected with cold $70 \%$ ethanol $\left(4^{\circ} \mathrm{C}\right)$ and finally stained with $1 \mathrm{ml}$ of propidium iodide (PI) $(20 \mathrm{mg} / \mathrm{ml})$ containing RNase $(1 \mathrm{mg} / \mathrm{ml})$ in PBS for $30 \mathrm{~min}$. Then the cells were detected with fluorescence activated cell sorting (FACS). For the apoptosis assay, the cells were washed with PBS $\left(4^{\circ} \mathrm{C}\right)$, incubated with PI and Annexin V-FITC following the instructions of the kits (KeyGen Biotech, Nanjing, China). Subsequently, the cells were analyzed by FACS.

Xenograft tumor model of human OS. The use and care of animals was approved by the Institutional Animal Care and Use Committee (IACUC) of Chongqing Medical University. Athymic nude mice (female, 4- 6 weeks old, 4 mice/group) were purchased from the Animal Center of Chongqing Medical University (Chongqing, China). Sub-confluent cells (143B) were washed and re-suspended in PBS $\left(4^{\circ} \mathrm{C}\right)$ to a density of $4 \times 10^{7}$ cells $/ \mathrm{ml}$ and then were subcutaneously injected into the right flanks of athymic nude mice using a $27-\mathrm{G}$ needle (50 $\mu \mathrm{l}$, $\sim 2 \times 10^{6}$ cells). One week after the injections, the mice were treated with pre-designated doses (40 and $80 \mathrm{mg} / \mathrm{kg}$ ) of TET or solvent by intragastric administration daily for 4 weeks. Then, the mice were sacrificed and samples were harvested for histological evaluation.

Protein harvest and western blot analysis (WB) assay. 143B cells were seeded into a 6-well plate and treated with predesignated concentrations (1, 2 and $4 \mu \mathrm{M})$ of TET, SB203580 or DMSO. At pre-designated time points, cells were washed with PBS $\left(4^{\circ} \mathrm{C}\right)$ and lysed with ice-cold lysis buffer $(300 \mu \mathrm{l})$. The lysates were boiled for $10 \mathrm{~min}$. After electrophoresis, proteins were transferred to polyvinylidene fluoride (PVDF) membranes, then blotted with corresponding primary antibodies and incubated with HRP-labeled secondary antibodies successively. The target proteins were developed with the SuperSignal West Pico Substrate (Pierce, Rockford, IL, USA). Each condition was carried out in triplicate.

Histological evaluation and immunohistochemical (IHC) staining. Retrieved tumor masses were fixed with paraformaldehyde (4\%) and embedded with paraffin, respectively. Serial sections were stained with hematoxylin and eosin (H\&E). For IHC staining, the processed slides were deparaffinized and rehydrated in a graduated manner. The deparaffinized sections were incubated with proliferating cell and nuclear antigen (PCNA) antibody (1:100 dilution) or PTEN antibody (1:100 dilution) or isotype $\mathrm{IgG}$ as a control. Finally, the sections were incubated with streptavidin-labeled secondary antibodies and then visualized with diaminobenzidine (DAB) staining.

Statistical analysis. The results of all experiments are presented as the mean \pm standard deviation (SD) of at least 3 independent tests. Statistical analysis of the results was conducted using a 
A

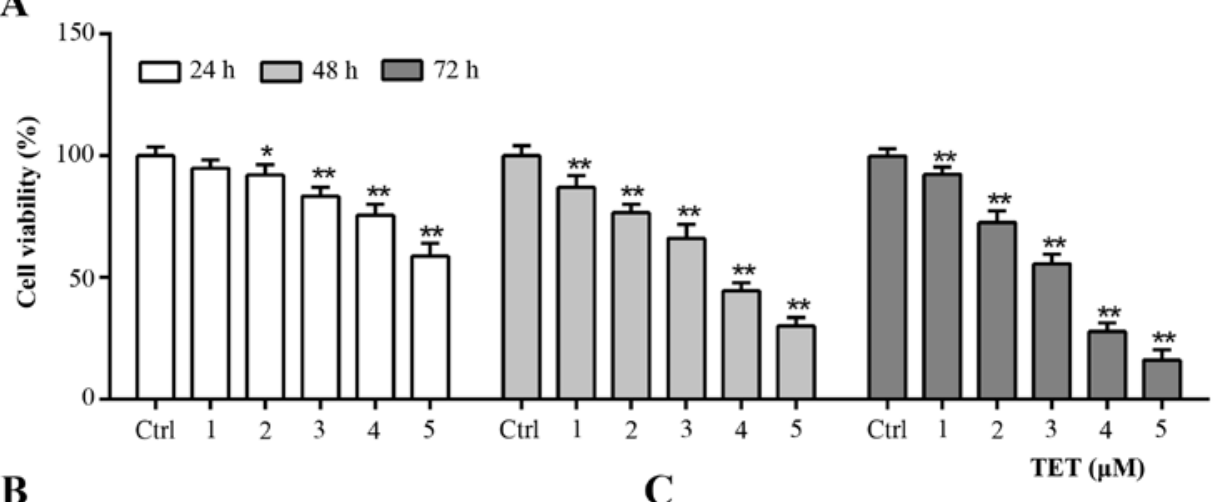

B

C

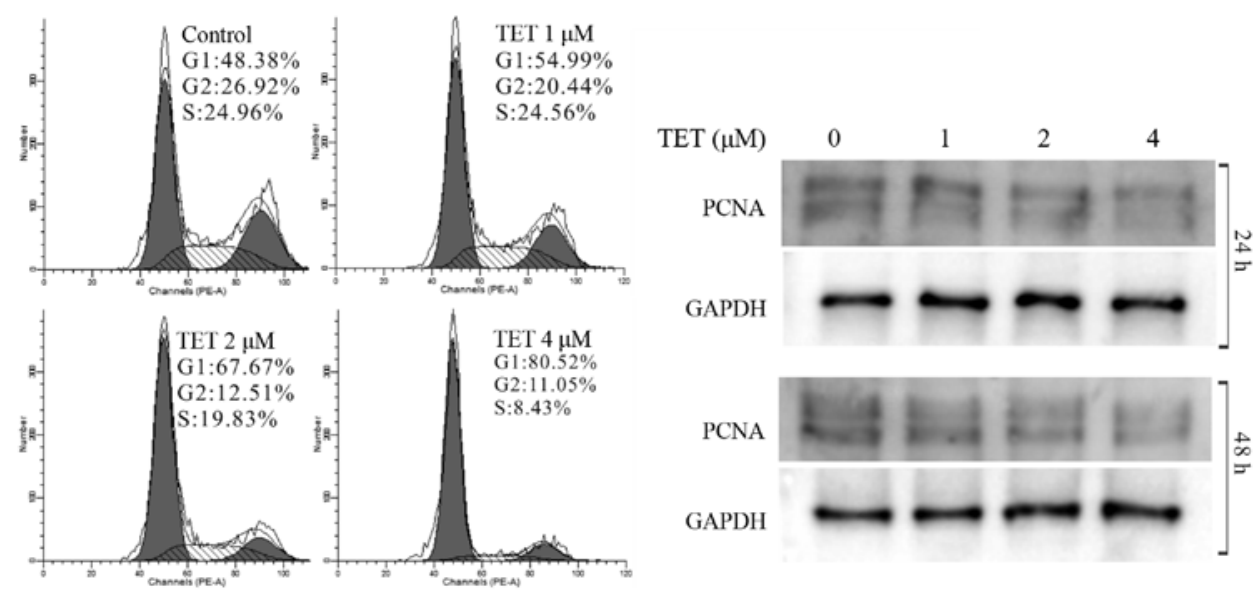

Figure 1. Effects of TET on the proliferation in 143B cells. (A) CCK-8 assay results revealed the antiproliferative effect of TET in $143 \mathrm{~B}$ cells ("P $<0.05$, compared with the control; ${ }^{* *} \mathrm{P}<0.01$, compared with the control). (B) Flow cytometric analysis demonstrated that TET induces cell cycle arrest in $143 \mathrm{~B}$ cells. (C) WB assay results revealed that TET affects the protein expression level of PCNA in 143B cells. TET, tetrandrine; WB, western blot analysis; PCNA, proliferating cell nuclear antigen.

t-test by GraphPad Prism 5 (GraphPad Software, Inc., La Jolla, CA, USA), and a P-value $<0.05$ was considered to indicate a statistically significant result.

\section{Results}

TET exhibits an antiproliferative effect and arrests the cell cycle at the G1 phase in 143B cells. To validate whether TET could be used as a chemotherapeutic agent for OS patients, CCK-8 assay was applied to explore the antiproliferative effect of TET on 143B cells. The results revealed that TET inhibited the proliferation of 143B cells effectively in a concentration- and time-dependent manner (Fig. 1A). According to the CCK-8 assay result, we chose 1,2 and $4 \mu \mathrm{M}$ of TET to perform the following experiments. Cell cycle analysis results revealed that TET induced cell cycle arrest at the G1 phase in 143B cells, and that the cell cycle arrest effect was positively related with the concentration of TET (Fig. 1B). The WB assay showed that TET significantly inhibited the expression of proliferating cell nuclear antigen (PCNA) (Fig. 1C). These data suggest that TET may be used as a chemotherapeutic agent or adjuvant for human OS treatment.

TET induces apoptosis in $143 \mathrm{~B}$ cells. We next conducted further analyses to demonstrate whether TET induced apoptosis of 143B cells. The results showed that TET increased the apoptotic cell rate concentration-dependently (Fig. 2A). WB revealed that TET substantially upregulated the protein level of Bad, but downregulated the protein level of Bcl-2 in the 143B cells (Fig. 2B). These results strongly indicate that TET is a potent apoptosis inducer in 143B cells.

TET inhibits human OS growth in a xenograft tumor model. To investigate the in vivo anticancer activity of TET, we applied a well-established xenograft OS model. Compared with the solvent control group, TET suppressed the tumor growth dose-dependently (Fig. 3A and B). Upon H\&E staining, more necrotic cells were found in the TET-treated tumors than this number in the control group, and more necrotic cells were found with the higher dose of TET (Fig. 3C). To investigate any potential cytotoxic effects of TET, we measured the weight of the kidney, spleen, liver and the mouse body weight for each group, and no statistical difference was found among the groups. In addition, liver and kidney sections were dealt with H\&E staining, and no obvious morphological change was discovered, indicating that the present dose of TET is nontoxic (Fig. 3B and C). IHC staining results showed that PCNA was dose-dependently downregulated by TET in OS (Fig. 3D). These results showed that TET can inhibit the proliferation of 143B cells in vivo and is relatively safe. These results further demonstrated that TET may be a potential anticancer agent for OS.

PTEN is involved in the antiproliferative effect of TET in $143 \mathrm{~B}$ cells. We investigated whether PTEN is involved in 
A

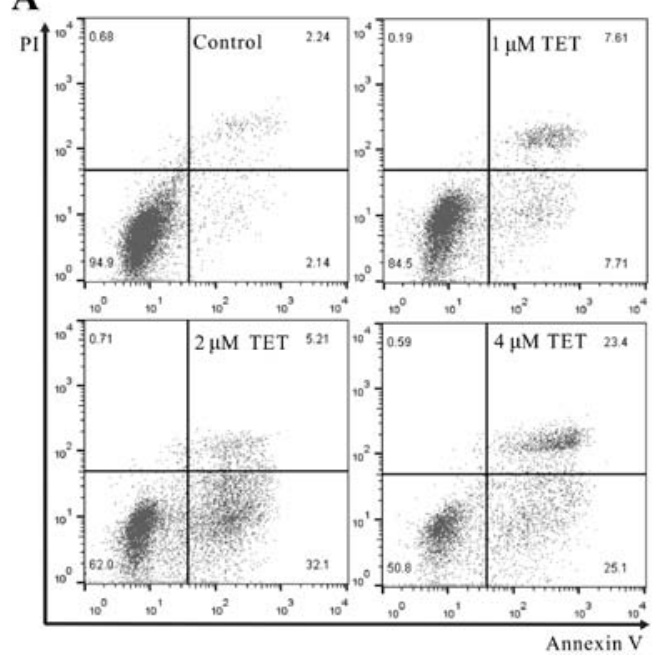

B

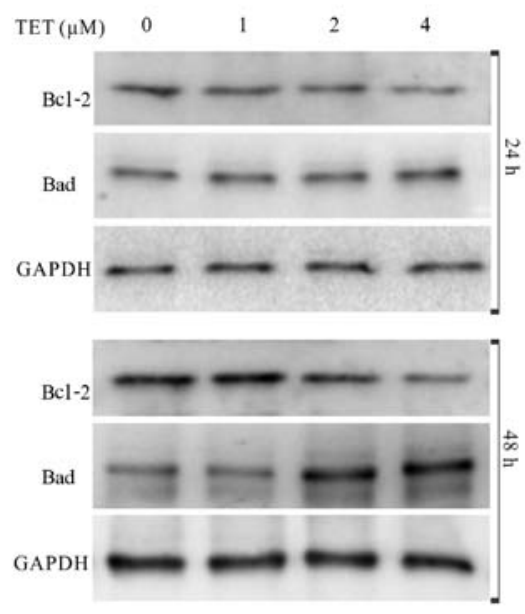

Figure 2. Effects of TET on the apoptosis of 143B cells. (A) Flow cytometric analysis showed that TET induced apoptosis in the 143B cells. (B) WB assay results revealed that TET affected the protein levels of Bad and Bcl-2 in the 143B cells. TET, tetrandrine; WB, western blot analysis.

A

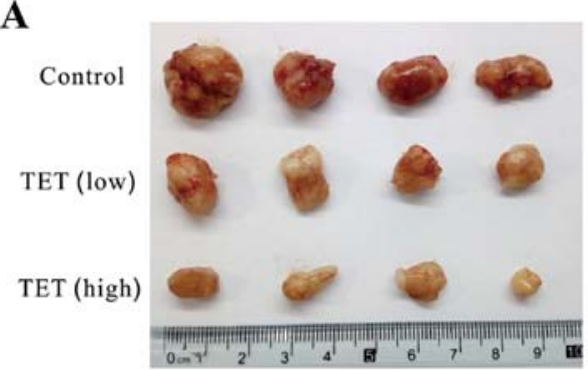

C

os

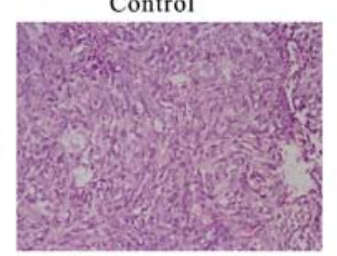

Liver

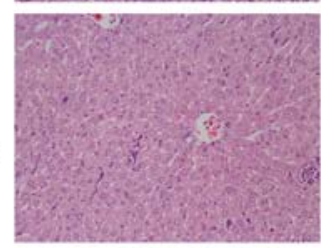

Kidney

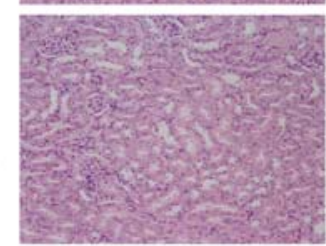

D

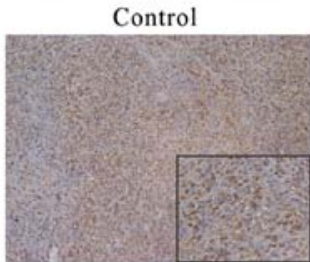

B

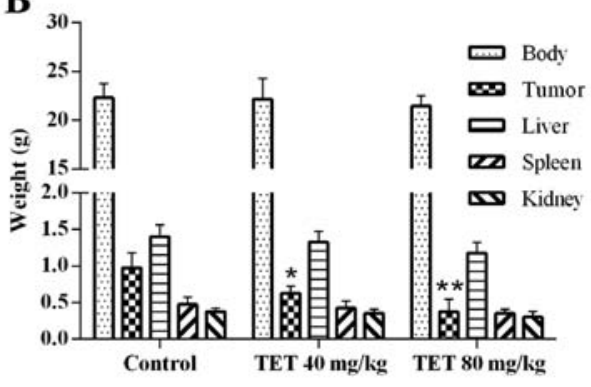

TET (low)
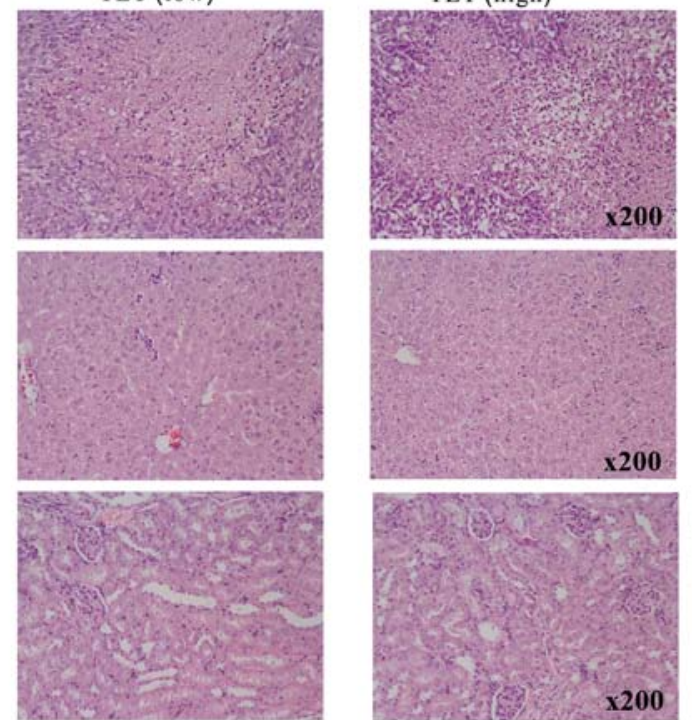

TET (low)

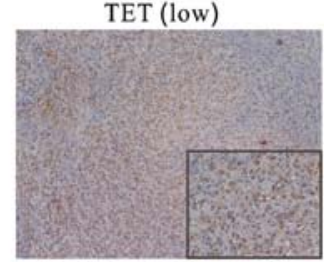

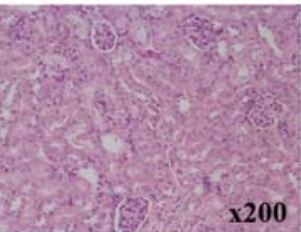

TET (high)

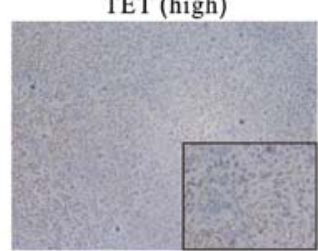

Figure 3. Effects of TET on the tumor growth of OS. (A) Tumor masses collected from nude mice revealed the anticancer effect of TET on OS. (B) Quantitative results of the weight of the kidney, spleen and liver, mouse body weight and tumor weight of each group showed that TET had an antiproliferative effect on OS without having toxic side-effects ( $\mathrm{P}<0.05$, compared with the control; ${ }^{* *} \mathrm{P}<0.01$, compared with the control). (C) H\&E staining results show the effect of TET on OS, liver and kidney samples; representative results are shown. (D) IHC staining show that TET affects the expression level of PCNA in OS; representative results are shown. TET, tetrandrine; OS, osteosarcoma; H\&E, hematoxylin and eosin; IHC, immunohistochemical; PCNA, proliferating cell nuclear antigen. 
A

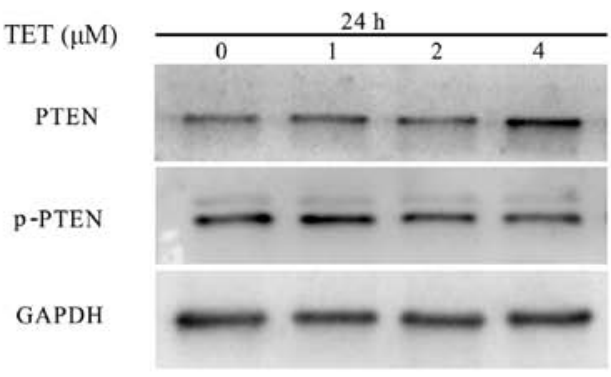

B

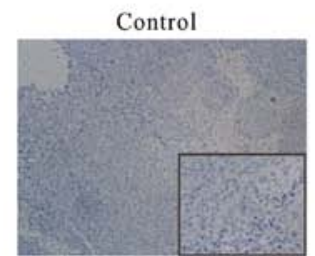

C

AdRFP

AdPTEN

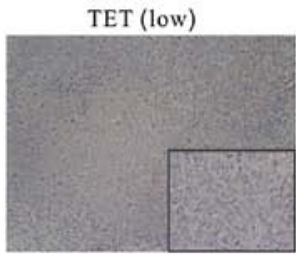

AdsiPTEN

$\mathrm{x} 100$

产

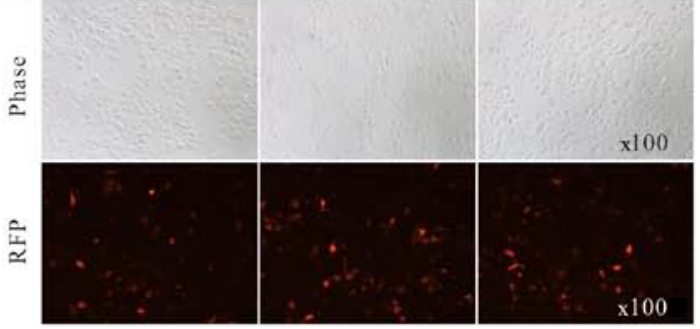

D

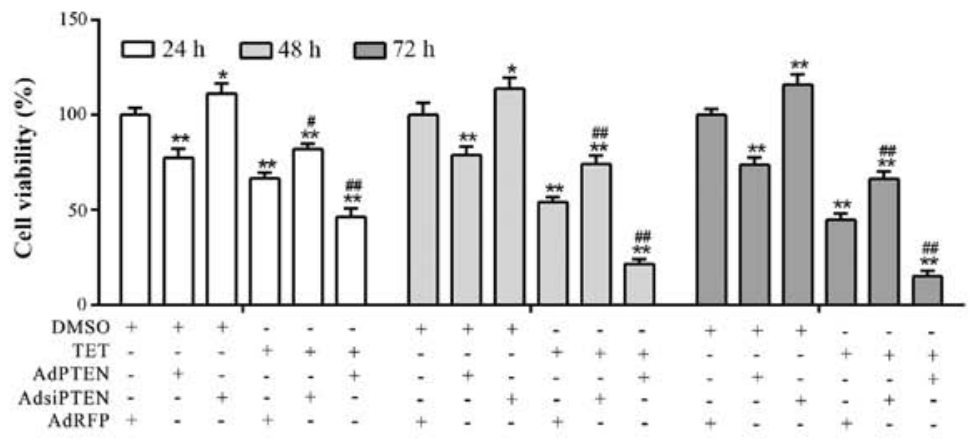

E
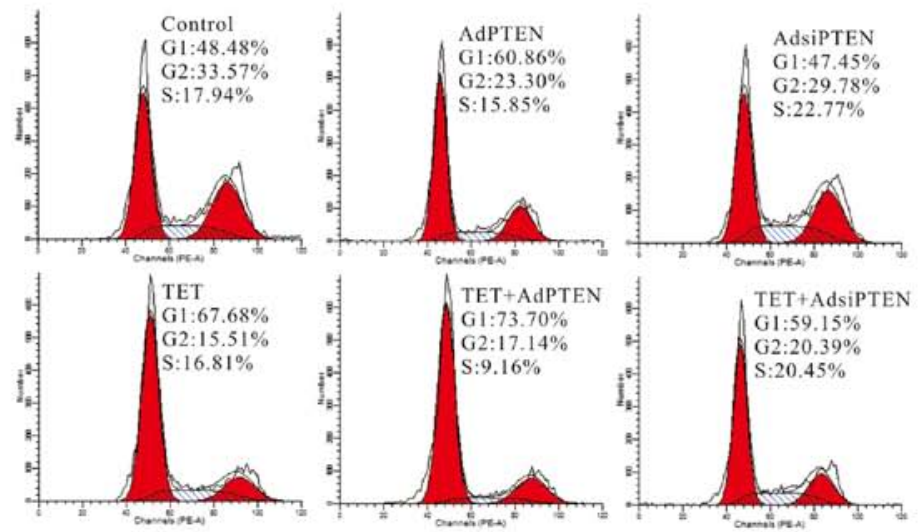

Figure 4. TET increases the level of PTEN in 143B cells. (A) WB assay results show that TET affected the expression level of PTEN and p-PTEN in 143B cells. (B) IHC staining results show that TET affected the expression level of PTEN in OS; representative results are shown. (C) Fluorescence images and WB assay results show the proper functioning of the recombinant adenoviruses in 143B cells. (D) CCK-8 assay results show that the TET-induced antiproliferative effect may be affected by exogenous expression of PTEN or knockdown of PTEN in 143B cells; the concentration of TET herein is $3 \mu \mathrm{M}$ ("P $<0.05$, compared with the control; ${ }^{* *} \mathrm{P}<0.01$, compared with the control; ${ }^{~} \mathrm{P}<0.05$, compared with TET; ${ }^{\# \#} \mathrm{P}<0.01$, compared with TET). (E) Flow cytometric analysis results of each group show how the TET-induced cell cycle arrest in 143B cells is affected by the expression level of PTEN. TET, tetrandrine; WB, western blot analysis; PTEN, phosphatase and tensin homologue; IHC, immunohistochemical; OS, osteosarcoma.

the antiproliferative effect of TET in 143B cells as in other cancer cells (23). The WB assay results showed that TET concentration- and time-dependently induced the expression of
PTEN (Fig. 4A). The IHC staining results revealed that PTEN was upregulated dose-dependently in vivo after treatment with TET (Fig. 4B). Before being used in the present study, 
A

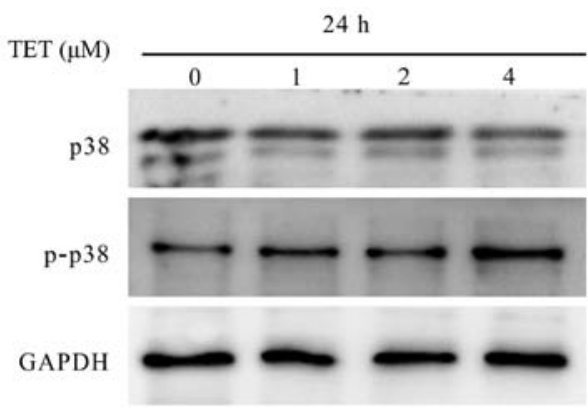

$48 \mathrm{~h}$

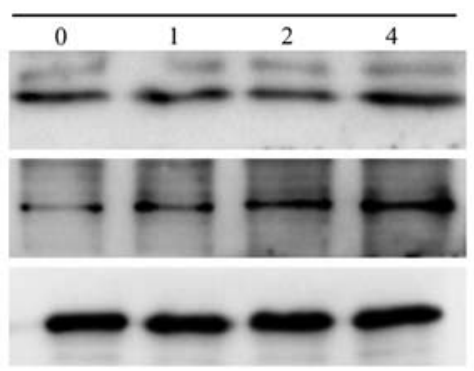

B
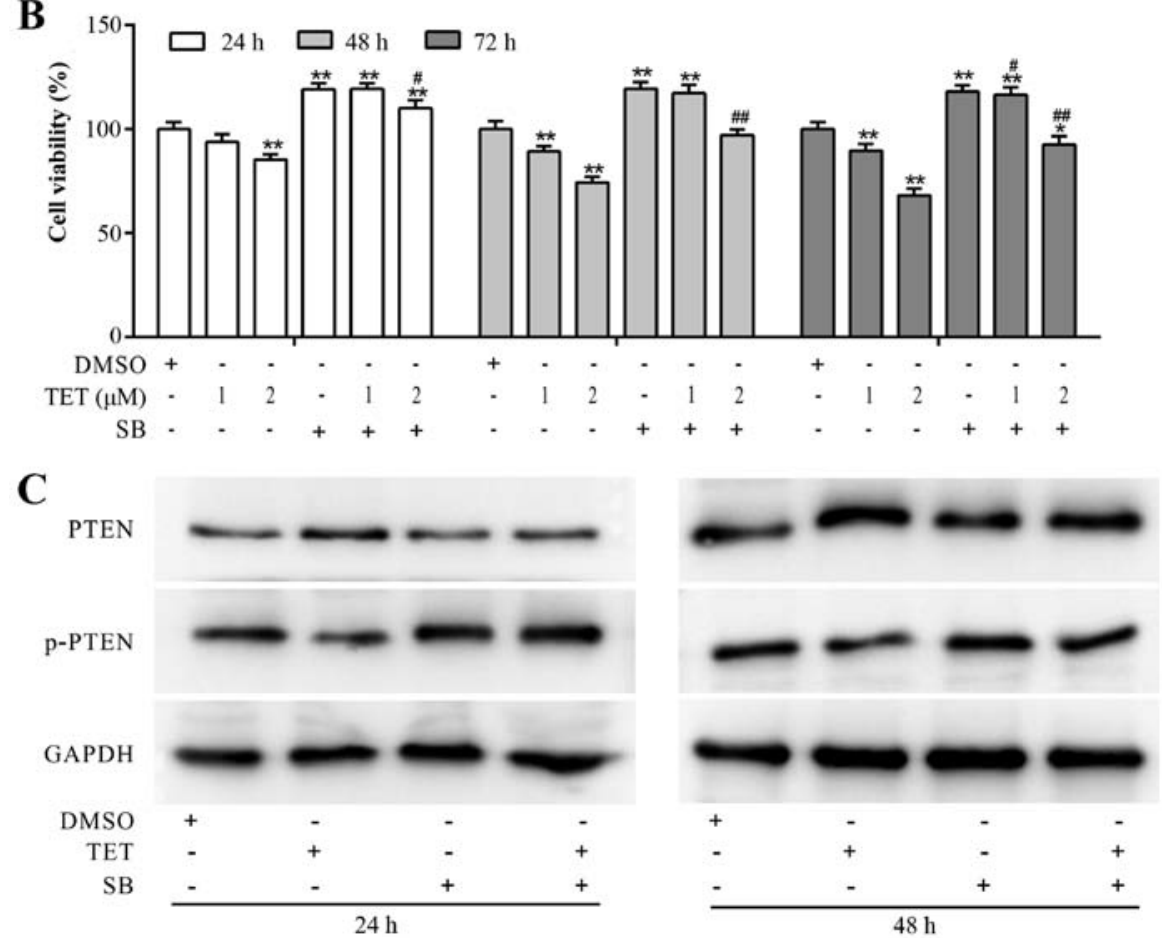

Figure 5. p38 MAPKs mediate the effect of TET on PTEN in 143B cells. (A) WB assay results show that TET affected the expression level of p38 and p-p38 in 143B cells. (B) CCK-8 results show that the TET-induced antiproliferative effect may be affected by p38 inhibitor SB203580 (SB, $0.6 \mu \mathrm{M}$ ) in $143 \mathrm{~B}$ cells $\left(\right.$ ( $\mathrm{P}<0.05$, compared with the control; ${ }^{* *} \mathrm{P}<0.01$, compared with the control; ${ }^{*} \mathrm{P}<0.05$, compared with $\mathrm{SB} ;{ }^{\# \#} \mathrm{P}<0.01$, compared with SB). (C) WB assay results show that TET combined with SB affects the expression level of p-PTEN and PTEN. TET, tetrandrine; PTEN, phosphatase and tensin homologue.

the recombinant adenoviruses were ascertained for proper functioning in 143B cells by fluorescence images and WB assay (Fig. 4C). The CCK-8 assay demonstrated that exogenous expression of PTEN substantially enhanced the antiproliferative effect of TET, while knockdown of PTEN decreased this effect of TET in the 143B cells (Fig. 4D). Moreover, flow cytometric analysis revealed that the TET-induced cell cycle arrest in 143B cells could be strengthened by the exogenous expression of PTEN, and weakened by the knockdown of PTEN (Fig. 4E). These results consequently indicated that PTEN is involved in the antiproliferative effect of TET in 143B cells.

TET upregulates PTEN by activating p38 MAPK in 143B cells. Although, PTEN partly mediates the antiproliferative effect of TET in 143B cells, the mechanism involved in the upregulation of PTEN by TET remains unknown. With further analysis, we found that TET concentration-dependently increased the phosphorylation of p38 MAPK (Fig. 5A). CCK-8 assay results demonstrated that the antiproliferative effect of TET was partly reversed by SB203580 (SB) in the 143B cells (Fig. 5B). When TET was combined with SB to treat 143B cells, the expression level of PTEN was decreased, and the expression level of p-PTEN was markedly increased (Fig. 5C). These results suggested that the upregulation of PTEN may have resulted from the activation of p38 MAPK, which may block the phosphorylation of PTEN in the 143B cells.

\section{Discussion}

In the present study, we demonstrated that TET may be a potential and effective antiproliferation drug for OS. Mechanistically, we discovered that the anticancer ability of TET may be partly mediated by upregulating PTEN signaling. Moreover, the upregulation of PTEN signaling is mediated by the activation of p38 MAPK.

As one of the most frequent primary malignant bone tumors that occurs in childhood, OS is conventionally treated with chemotherapy agents. However, the chemotherapy drugs used currently do not only exhibit low efficiency, but also produce numerous side-effects $(6,24)$. Thus, it is of high significance to take notice of new drug research and developments, so as to improve the quality of life of patients. TET is 
a bisbenzylisoquinoline alkaloid which is extracted from the dried root of Chinese herb medicinal plant Hang Fang Ji. In addition, recent studies have shown no side-effects of TET in the treatment of silicosis and lung cancer (25), which makes it an ideal anticancer drug. Moreover, with definite knowledge on the synthesis, distribution, extraction, structural elucidation and pharmacological properties of TET, this very herb has been confirmed as a potential agent capable of inhibiting proliferation and inducing apoptosis in many malignant cancer cells $(12,25)$. In the present study, we found that TET has antiproliferative and apoptosis-inducing effects in 143B cells. Moreover, TET has been reported to have the ability to arrest the cell cycle at different phases in various cancer cells $(9,13,26)$. In our research, TET arrested the cell cycle at the G1 phase in 143B cells. Moreover, TET inhibited human OS growth in a xenograft tumor model. Thus, it is highly suggested that TET could be used as a potential anticancer drug for human OS.

The effect of the pharmacological property of TET on malignant cancer cells has been confirmed to be achieved through different pathways such as the calcium channel (27), reactive oxygen species (28), caspase-dependent pathway (29), reversal of multidrug resistance (30), decrease of phosphorylated Akt (31) and activation of Jnk1/2 (32). For many malignant cancers, such as prostate cancer, the mutation of PTEN plays an essential role (33) and overexpression of PTEN results in a higher chemosensitivity (34) which indicates that PTEN is a tumor-suppressor gene. Thus, PTEN may be a potential target for OS treatment. However, this concept warrants further study. Therefore, we investigated whether PTEN is involved in the antiproliferative effect of TET in 143B cells. As a lipid phosphatase catalyzing $\mathrm{PIP}_{3}$ dephosphorylation resulting in the production of $\mathrm{PIP}_{2}, \mathrm{PTEN}$ inhibits the activation of the PI3K/Akt/mTOR pathway, which is implicated in many cellular functions including cell proliferation, survival and inhibition of apoptosis (35). Moreover, the phosphorylation of PTEN inactivates PTEN from its anticancer ability, which means the ratio of p-PTEN/PTEN is positively related with the inactivation level of PTEN (36). In the present study, TET increased the protein level of PTEN and decreased the level of p-PTEN. Exogenous expression of PTEN substantially enhanced the antiproliferation and cell cycle arrest produced by TET, while knockdown of PTEN partly reduced this effect of TET in 143B cells. Hence, we speculated that TET may increase the protein level of PTEN as well as decrease its phosphorylation. Although, the precise mechanism underlying this process remains unclear, we have reason to speculate that the antiproliferative and apoptosis-inducing effect of TET is related with the PTEN pathway.

As a subgroup of the MAPKs, p38 was first described as a transducer of the response to environmental stress conditions and as a critical mediator of inflammatory cytokines. Subsequently, p38 was demonstrated to have the ability to regulate different processes including cell cycle, differentiation, inflammation, senescence, autophagy and apoptosis (37). p38 MAPKs are phosphorylated and activated by MAPK kinases. Once activated, p38 MAPKs phosphorylate the serine/threonine residues of their substrates, which include several transcription factors as well as protein kinases (38). It has been reported that the activation and phosphorylation of p38 MAPK mediates the anticancer activity in numerous malignant cancers such as pancreatic (39) and gastric cancer (40), and head and neck carcinoma (41).

We hypothesized that in OS, p38 MAPK may be associated with PTEN in a direct or indirect way. In the present study, TET activated and phosphorylated p38 MAPK in 143B cells, while upregulating PTEN and decreasing the level of p-PTEN. In addition the p38 MAPK inhibitor was partly capable of reversing the TET-induced antiproliferation in 143B cells, the upregulation of PTEN and the downregulation of p-PTEN. Consequently, it is strongly suggested that the upregulation of PTEN by TET may be the result of the decrease in p-PTEN, which may be mediated by the TET-induced activation and phosphorylation of $\mathrm{p} 38$ MAPK.

In summary, we demonstrated that TET can be used as an effective chemotherapeutic agent for human OS. The anticancer activity of TET in 143B cells may be mediated by an increase in PTEN, which may result from the TET-induced activation of p38 MAPK. Future studies should be carried out to investigate the possible molecular mechanism of TET on the activation of p38 MAPK, and elucidate the possible interaction between p38 MAPK and PTEN phosphorylation in 143B cells. Furthermore, as the present study did not involve the migration and metastasis of OS cells, a number of subsequent experiments and pre-clinical assessments should be carried out for further evaluation of this drug.

\section{Acknowledgements}

The present study was supported in part by grants from the National Natural Science Foundation of China (grants no. 81672230), and the Natural Science Foundation of Chongqing (grant no. cstc2013jjB10021). Sincerely, we thank Dr T.C. He (University of Chicago Medical Center, USA) for his generous gift of all recombinant adenoviruses.

\section{References}

1. Wan G, Tao JG, Wang GD, Liu SP, Zhao HX and Liang QD: In vitro antitumor activity of the ethyl acetate extract of Potentilla chinensis in osteosarcoma cancer cells. Mol Med Rep 14: 36343640, 2016.

2. Meng ZJ, Wu N, Liu Y, Shu KJ, Zou X, Zhang RX, Pi CJ, He BC, Ke ZY, Chen L, et al: Evodiamine inhibits the proliferation of human osteosarcoma cells by blocking PI3K/Akt signaling. Oncol Rep 34: 1388-1396, 2015.

3. Scholten DJ II, Timmer CM, Peacock JD, Pelle DW, Williams BO and Steensma MR: Down regulation of Wnt signaling mitigates hypoxia-induced chemoresistance in human osteosarcoma cells. PLoS One 9: e111431, 2014.

4. Pruksakorn D, Teeyakasem P, Klangjorhor J, Chaiyawat P, Settakorn J, Diskul-Na-Ayudthaya P, Chokchaichamnankit D, Pothacharoen P and Srisomsap C: Overexpression of KH-type splicing regulatory protein regulates proliferation, migration, and implantation ability of osteosarcoma. Int J Oncol 49: 903-912, 2016.

5. Ma K, Huang MY, Guo YX and Hu GQ: Matrine-induced autophagy counteracts cell apoptosis via the ERK signaling pathway in osteosarcoma cells. Oncol Lett 12: 1854-1860, 2016.

6. Sarman H, Bayram R and Benek SB: Anticancer drugs with chemotherapeutic interactions with thymoquinone in osteosarcoma cells. Eur Rev Med Pharmacol Sci 20: 1263-1270, 2016.

7. Wei X, Qu TL, Yang YF, Xu JF, Li XW, Zhao ZB and Guo YW: Design and synthesis of new tetrandrine derivatives and their antitumor activities. J Asian Nat Prod Res 18: 966-975, 2016.

8. Liu T, Liu X and Li W: Tetrandrine, a Chinese plant-derived alkaloid, is a potential candidate for cancer chemotherapy. Oncotarget 7: 40800-40815, 2016. 
9. Lei RR, Hu HF, Bai F, Liu Y, Wu CZ, Huang XX, Xie LP and $\mathrm{Hu}$ YJ: Anti-proliferative and apoptotic effects of S1, a tetrandrine derivative, in human gastric cancer BGC-823 cells. Chin J Nat Med 14: 527-533, 2016.

10. Zhang Y, Liu W, He W, Zhang Y, Deng X, Ma Y, Zeng J and Kou B: Tetrandrine reverses epithelial-mesenchymal transition in bladder cancer by downregulating Gli-1. Int J Oncol 48: 2035-2042, 2016.

11. Li D, Lu Y, Sun P, Feng LX, Liu M, Hu LH, Wu WY, Jiang BH, Yang M, Qu XB, et al: Inhibition on proteasome $\beta 1$ subunit might contribute to the anti-cancer effects of fangchinoline in human prostate cancer cells. PLoS One 10: e0141681, 2015.

12. Wu K, Zhou M, Wu QX, Yuan SX, Wang DX, Jin JL, Huang J, Yang JQ, Sun WJ, Wan LH, et al: The role of IGFBP-5 in mediating the anti-proliferation effect of tetrandrine in human colon cancer cells. Int J Oncol 46: 1205-1213, 2015.

13. Zhu R, Liu T, Tan Z, Wu X, Li M, Jiang L, Bao R, Shu Y, Lu A and Liu Y: Tetrandrine induces apoptosis in gallbladder carcinoma in vitro. Int J Clin Pharmacol Ther 52: 900-905, 2014.

14. Tian Y, Yin H and Xu H: Enhanced pro-apoptotic effect of tetrandrine loaded nanoparticles against osteosarcoma cells. Curr Drug Deliv 13: 946-952, 2016.

15. Tao LJ, Zhou XD, Shen CC, Liang CZ, Liu B, Tao Y and Tao HM Tetrandrine induces apoptosis and triggers a caspase cascade in U2-OS and MG-63 cells through the intrinsic and extrinsic pathways. Mol Med Rep 9: 345-349, 2014.

16. Shojaee S, Chan LN, Buchner M, Cazzaniga V, Cosgun KN, Geng H, Qiu YH, von Minden MD, Ernst T, Hochhaus A, et al: $P T E N$ opposes negative selection and enables oncogenic transformation of pre-B cells. Nat Med 22: 379-387, 2016.

17. Liu YL and Yan Y: Timing of the loss of Pten protein determines disease severity in a mouse model of myeloid malignancy. 127: 1912-1922, 2016

18. Johnson GL and Lapadat R: Mitogen-activated protein kinase pathways mediated by ERK, JNK, and p38 protein kinases. Science 298: 1911-1912, 2002.

19. Banerjee B, Nandi P, Chakraborty S, Raha S, Sen PC and Jana K: Resveratrol ameliorates benzo(a)pyrene-induced testicular dysfunction and apoptosis: Involvement of p38 MAPK/ATF2/ iNOS signaling. J Nutr Biochem 34: 17-29, 2016

20. Li J, Zhang J, Tang M, Xin J, Xu Y, Volk A, Hao C, Hu C, Sun J, Wei W, et al: Hematopoietic stem cell activity is regulated by Pten phosphorylation through a niche-dependent mechanism. Stem Cells 34: 2130-2144, 2016.

21. Wu QX, Yuan SX, Ren CM, Yu Y, Sun WJ, He BC and Wu K Oridonin upregulates PTEN through activating p38 MAPK and inhibits proliferation in human colon cancer cells. Oncol Rep 35 $3341-3348,2016$

22. Luo J, Deng ZL, Luo X, Tang N, Song WX, Chen J, Sharff KA, Luu HH, Haydon RC, Kinzler KW, et al: A protocol for rapid generation of recombinant adenoviruses using the AdEasy system. Nat Protoc 2: 1236-1247, 2007.

23. Rhei E, Kang L, Bogomolniy F, Federici MG, Borgen PI and Boyd J: Mutation analysis of the putative tumor suppressor gene PTEN/MMACl in primary breast carcinomas. Cancer Res 57 3657-3659, 1997.

24. Wang L, Wang W, Rui Z and Zhou D: The effective combination therapy against human osteosarcoma: Doxorubicin plus curcumin co-encapsulated lipid-coated polymeric nanoparticulate drug delivery system. Drug Deliv 23: 3200-3208, 2016.

25. Bhagya $\mathrm{N}$ and Chandrashekar KR: Tetrandrine - A molecule of wide bioactivity. Phytochemistry 125: 5-13, 2016.

26. Xiao W, Jiang Y, Men Q, Yuan L, Huang Z, Liu T, Li W and Liu X: Tetrandrine induces G1/S cell cycle arrest through the ROS/Akt pathway in EOMA cells and inhibits angiogenesis in vivo. Int J Oncol 46: 360-368, 2015.
27. Chiou WF, Lee WS and Yeh PH: Tetrandrine selectively protects against amyloid-beta protein - but not against MPTP-induced cytotoxicity in SK-N-SH neuroblastoma cells. Planta Med 72: 1300-1304, 2006

28. Shen YC, Chen CF, Wang SY and Sung YJ: Impediment to calcium influx and reactive oxygen production accounts for the inhibition of neutrophil Mac-1 Up-regulation and adhesion by tetrandrine. Mol Pharmacol 55: 186-193, 1999.

29. Oh SH and Lee BH: Induction of apoptosis in human hepatoblastoma cells by tetrandrine via caspase-dependent Bid cleavage and cytochrome $c$ release. Biochem Pharmacol 66: 725-731, 2003.

30. Xu M, Sheng LH,Zhu XH,Zeng SB and Zhang GJ: Reversal effect of Stephania tetrandra-containing Chinese herb formula SENL on multidrug resistance in lung cancer cell line SW1573/2R120. Am J Chin Med 38: 401-413, 2010.

31. Xing ZB, Yao L, Zhang GQ, Zhang XY, Zhang YX and Pang D: Fangchinoline inhibits breast adenocarcinoma proliferation by inducing apoptosis. Chem Pharm Bull 59: 1476-1480, 2011.

32. Chaudhary $\mathrm{P}$ and Vishwanatha JK: c-Jun $\mathrm{NH}_{2}$-terminal kinase-induced proteasomal degradation of c-FLIP ${ }_{\mathrm{L} / \mathrm{S}}$ and $\mathrm{Bcl}_{2}$ sensitize prostate cancer cells to Fas- and mitochondria-mediated apoptosis by tetrandrine. Biochem Pharmacol 91: 457-473, 2014.

33. Fallahabadi ZR, Noori Daloii MR, Mahdian R, Behjati F, Shokrgozar MA, Abolhasani M, Asgari M and Shahrokh H: Frequency of PTEN alterations, TMPRSS2-ERG fusion and their association in prostate cancer. Gene 575: 755-760, 2016.

34. Liang N, Zhou X, Zhao M, Zhao D, Zhu Z, Li S and Yang H: Down-regulation of microRNA-26b modulates non-small cell lung cancer cells chemoresistance and migration through the association of PTEN. Acta Biochim Biophys Sin 47: 530-538, 2015.

35. Mi S, Xiang G, Yuwen D, Gao J, Guo W, Wu X, Wu X, Sun Y, $\mathrm{Su} \mathrm{Y}$, Shen Y, et al: Inhibition of autophagy by andrographolide resensitizes cisplatin-resistant non-small cell lung carcinoma cells via activation of the Akt/mTOR pathway. Toxicol Appl Pharmacol 310: 78-86, 2016.

36. Xiong J, Li Z, Zhang Y, Li D, Zhang G, Luo X, Jie Z, Liu Y, Cao Y, Le Z, et al: PRL-3 promotes the peritoneal metastasis of gastric cancer through the PI3K/Akt signaling pathway by regulating PTEN. Oncol Rep 36: 1819-1828, 2016.

37. Šrámek J, Němcová-Fürstová V and Kováŕ J: Kinase signaling in apoptosis induced by saturated fatty acids in pancreatic $\beta$-cells. Int J Mol Sci 17: 17, 2016.

38. Segalés J, Perdiguero E and Muñoz-Cánoves P: Regulation of muscle stem cell functions: A focus on the p38 MAPK signaling pathway. Front Cell Dev Biol 4: 91, 2016.

39. Wang L, Bai YY, Yang Y, Hu F, Wang Y, Yu Z, Cheng Z and Zhou J: Diabetes mellitus stimulates pancreatic cancer growth and epithelial-mesenchymal transition-mediated metastasis via a p38 MAPK pathway. Oncotarget 7: 38539-38550, 2016.

40. Su CC, Chen JY, Din ZH, Su JH, Yang ZY, Chen YJ, Wang RY and Wu YJ: 13-acetoxysarcocrassolide induces apoptosis on human gastric carcinoma cells through mitochondria-related apoptotic pathways: p38/JNK activation and PI3K/AKT suppression. Mar Drugs 12: 5295-5315, 2014.

41. Benvenuto M, Mattera R, Masuelli L, Taffera G, Andracchio O, Tresoldi I, Lido P, Giganti MG, Godos J, Modesti A, et al: $( \pm)$-Gossypol induces apoptosis and autophagy in head and neck carcinoma cell lines and inhibits the growth of transplanted salivary gland cancer cells in BALB $/ c$ mice. Int J Food Sci Nutr: $1-15,2016$. 